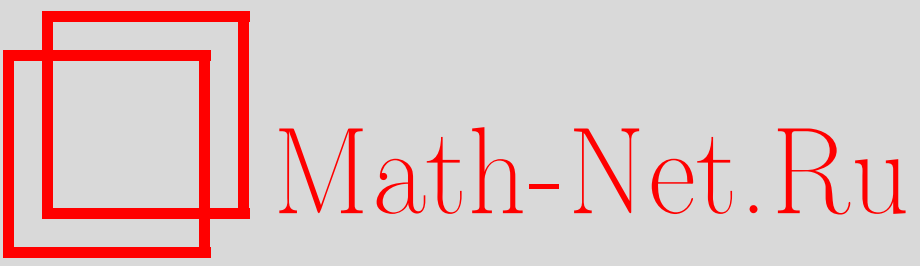

Н. А. Тюрин, Теорема существования для многообразия модулей борзоммерфельдовых лагранжевых циклов, УМH, 2005, том 60, выпуск 3, 179-180

DOI: https://doi.org/10.4213/rm1436

Использование Общероссийского математического портала Math-Net.Ru подразумевает, что вы прочитали и согласны с пользовательским соглашением

http://www.mathnet.ru/rus/agreement

Параметры загрузки:

IP: 54.162.27.143

26 апреля 2023 г., 18:29:22 


\title{
ТЕОРЕМА СУЩЕСТВОВАНИЯ ДЛЯ МНОГООБРАЗИЯ МОДУЛЕЙ БОР-ЗОММЕРФЕЛЬДОВЫХ ЛАГРАНЖЕВЫХ ЦИКЛОВ
}

\author{
Н. А. Тюрин
}

Настоящая короткая заметка посвящена вопросу существования бор-зоммерфельдовых лагранжевых циклов данного топологического типа. Напомним в двух словах общую постановку вопроса.

Многообразия модулей полувзвешенных бор-зоммерфельдовых лагранжевых циклов фиксированного топологического типа и объема были введены в работе [1]. Для произвольного компактного односвязного симплектического многообразия $(M, \omega)$ с целочисленной симплектической формой (т.е. $\left.[\omega] \in H^{2}(M, \mathbb{Z})\right)$ такое многообразие модулей $\mathscr{B}_{S}^{h w, r}$ состоит из пар $(S, \theta)$, где $S$ - бор-зоммерфельдов лагранжев цикл фиксированного топологического типа, а $\theta$-некоторый полувес на нем фиксированного объема. Стандартное условие лагранжевости (см., например, [2]) может быть дополнено условием Бора-Зоммерфельда относительно данных предквантования (см. [1]), которые представлены парой $(L, a)$, где $L$ - линейное комплексное расслоение, $a$ - эрмитова связность на $L$ такие, что $c_{1}(L)=[\omega], F_{a}=2 \pi i \omega$. В [1] определения и конструкции были объединены в новьй предмет, названньй алгебраической лагранжевой геометрией. Он оказался весьма полезньм в задаче геометрического квантования (см. [3], [4]), однако одним из открытых вопросов остался вопрос существования бор-зоммерфельдовых лагранжевых циклов. Ситуация похожа на ту, что возникла вокруг многообразия модулей инстантонов (см. [5]), когда построенная локальная теория была дополнена результатом К. Таубса, утверждавшим существование по меньшей мере одной гладкой точки многообразия модулей инстантонов. В случае многообразия модулей бор-зоммерфелшдовых лагранжевых циклов локалшная теория, построенная в [1], должна быть дополнена подобной теоремой существования, иначе многообразия модулей могут оказаться виртуальными.

Цель настоящей заметки - доказательство следующего утверждения.

Теорема. Пусть $(M, \omega)$ - компактное односвязное $2 n$-мерное симплектическое многообразие с челочисленной формой $\omega$. Пусть класс гомологий срединной размерности $[S] \in H_{n}(M, \mathbb{Z})$ реализуется ориентируемым лагранжевым подмногообразием фиксированного топологического типа $S$. Тогда найдется такое натуральное число $k \in \mathbb{Z}_{+}$, что для любого $k^{\prime} \geqslant k$ многообразие модулей $\mathscr{B}_{S, k^{\prime}}$ бор-зоммерфельдовых относительно данных предквантования $\left(L^{k^{\prime}}, a_{k^{\prime}}\right)$ лагранжевых чиклов топологического типа $S$ непусто.

Простьм следствием этого утверждения будет непустота и многообразия модулей полувзвешенных бор-зоммерфелшдовых лагранжевых циклов $\mathscr{B}_{S, k}^{h w, r}$ в тех же предположениях, доказательство чего мы опускаем из-за его очевидности.

ДокАЗАТЕЛЬСтво теОРемЫ. Пусть $S \subset M$ - ориентируемый гладкий лагранжев цикл, представляющий класс $[S] \in H_{n}(M, \mathbb{Z})$, а $(L, a)$ - данные предквантования. Тогда (см. [1]) ограничение $\left.(L, a)\right|_{S}$ является топологически тривиальным расслоением с плоской связностью. Если характер $\chi_{a}(S): \pi_{1}(S) \rightarrow U(1)$, индуцируемый этой связностью, тривиален, то тогда $S$ сам по себе является бор-зоммерфельдовым лагранжевым циклом (см. [1]). Пусть этот характер нетривиален:

$$
\chi_{a}(S) \neq 1 \text {. }
$$

Тогда рассмотрим окрестность Дарбу-Вейнстейна лагранжева цикла $S$, т.е. такую трубчатую окрестность $N(S) \subset M$, что существует симплектоморфизи

$$
\phi_{D W}:\left(N(S),\left.\omega\right|_{N(S)}\right) \rightarrow\left(N_{\varepsilon}\left(T^{*} S\right), d \rho\right)
$$

где $N_{\varepsilon}\left(T^{*} S\right)$ есть $\varepsilon$-окрестность нулевого сечения кокасательного расслоения $T^{*} S$, а $d \rho$-каноническая симплектическая форма на $T^{*} S$ (см. [6]). Тогда, согласно теореме Дарбу-Вейнстейна

\footnotetext{
Работа выполнена при поддержке РФФИ (гранты № 05-01-00455 и 05-01-01086).
} 
(см. там же), лагранжевы подмногообразия, содержащееся в $N(S)$, под действием $\phi_{D W}$ отображаются в графоки замкнутых 1-форм на $S$ в $T^{*} S$. Используя $\phi_{D W}$ в обратную сторону, можно строить лагранжевы подмногообразия в $M$, достаточно близкие к $S$. При этом нетрудно видеть, что если самому подмногообразию $S$ соответствует характер $\chi_{a}(S)$ относительно данньх предквантования $(L, a)$, то для лагранжева подмногообразия $S_{1}=\phi_{D W}^{-1}(\tau)$, индуцируемого замкнутой 1-формой $\tau \in Z_{S}^{1}$, такого, что график $\Gamma_{\tau} \in N_{\varepsilon}\left(T^{*} S\right)$, такой характер будет иметь вид

$$
\begin{gathered}
\chi_{a}\left(S_{1}\right): \pi_{1}\left(S_{1}\right)=\pi_{1}(S) \rightarrow U(1), \\
\chi_{a}\left(S_{1}\right)=\chi_{a}(S)+\exp \left(2 \pi i \int_{\phi_{D W}(\gamma)} \tau\right),
\end{gathered}
$$

где $\gamma$ - ориентированная петля, представляющая соответствующий класс из $\pi_{1}\left(S_{1}\right)$ (cp. [1]).

Нетрудно видеть, что для любого $\chi_{a}(S) \in U(1)$ найдется замкнутая 1-форма $\tau$ такая, что соответствующее отображение $\chi_{a}(S)+\exp \left(2 \pi i \int_{\phi_{D W}(\gamma)} \tau\right)$ будет тривиально на $\pi_{1}(S)$. Проблема заключается в том, что такая форма $\tau$ может не удовлетворять условию $\Gamma_{\tau} \in N_{\varepsilon}\left(T^{*} S\right)$ (т.е. $\varepsilon$ может оказаться слишком малым, чтобы можно было скомпенсировать характер $\chi_{a}(S)$ ).

Существование окрестности $N(S)$ Дарбу-Вейнстейна позволяет определить следующее подмножество в пространстве всех характеров:

$$
\Delta \subset \Xi=\left\{\chi: \pi_{1}(S) \rightarrow U(1)\right\},
$$

а именно:

$$
\Delta\left(S, \phi_{D W}\right)=\left\{\chi=\exp \left(2 \pi i \int_{\phi_{D W}(\cdot)} \tau\right) \mid \Gamma_{\tau} \in N_{\varepsilon}\left(T^{*} S\right), \tau \in Z_{S}^{1}\right\} .
$$

Нетрудно видеть, что $\Delta$ есть стягиваемое подмножество в якобиане $\Xi=H^{1}(S, \mathbb{R}) / H^{1}(S, \mathbb{Z})$, расположенное вокруг нуля.

С другой стороны, при переходе к данным предквантования уровня $k$ :

$$
\left(L^{k}, a_{k}\right), \quad c_{1}\left(L^{k}\right)=k \omega, \quad F_{a_{k}}=2 \pi i k \omega,
$$

характер, индуцируемый на лагранжевом подмногообразии $S$, изменяется следующим образом:

$$
\chi_{a_{k}}(S)=\left(\chi_{a}(S)\right)^{1 / k},
$$

где под последним выражением имеется в виду примитивный корень $k$-й степени. Отсюда видно, что найдется такое натуральное число $k \in \mathbb{Z}_{+}$, что для любого $k^{\prime} \geqslant k$

$$
\chi a_{k^{\prime}}(S) \in \Delta\left(S, \phi_{D W}\right) \text {. }
$$

Отсюда следует, что для каждого $k^{\prime} \geqslant k$ характер $\chi a_{k^{\prime}}(S)$ может быть скомпенсирован достаточно малой замкнутой формой, а значит, утверждение теоремы 1 доказано.

Заметим, что приведенные выше несложные рассуждения не отвечают на вопрос о существовании многообразия модулей бор-зоммерфелшдовых циклов "вообще", а толњко сводят проблему существования $\mathscr{B}_{S}$ и $\mathscr{B}_{S}^{h w, r}$ к стандартной проблеме симплектической геометрии и классической механики - проблеме отыскания лагранжева подмногообразия.

\section{СПИСОК ЛИТЕРАТУРЫ}

[1] А. Л. Городенцев, А. Н. Тюрин // Изв. АН. Сер. матем. 2001. Т. 65. № 3. С. 15-50. [2] В.И. Арнольд, А.Б. Гивенталь. Симплектическая геометрия. М.: Мир, 1987. [3] Н. А. Тюрин // Изв. АН. Сер. матем. 2001. Т. 65. № 4. С. 191-204. [4] Н. А. Тюрин // Изв. АН. Сер. матем. 2002. Т. 66. № 3. С. 175-196. [5] S. Donaldson, P. Kronheimer. The Geometry of Four-manifolds. Oxford: Clarendon Press, 1990. [6] A. Weinstein // Ann. of Math. (2). 1973. V. 98. P. 377-410.

Лаборатория теоретической физики им. Н. Н. Боголюбова Объединенньй институт ядерных исследований (Дубна), Московский государственньй университет путей сообщения
Представлено А. Г. Сергеевьм Принято редколлегией 23.03.2005 